\title{
ESTIMATES OF LOGARITHMIC COEFFICIENTS OF UNIVALENT FUNTIONS
}

STEPHEN M. ZEMYAN

\author{
Department of Mathematics \\ The Pennsylvania State University \\ Mont Alto Campus \\ Mont Alto, Pennsylvania 17237-9703
}

(Received November 7, 1991 and in revised form June 19, 1992)

ABSTRACT. In this paper, we derive some consequences of Milin's inequality for the logarithmic coefficients of a univalent function by exploiting a reformulation of it.

KEY WORDS AND PHRASES. Univalent functions, logarithmic coefficients, Milin's inequality, convex weights.

1991 MATHEMATICS SUBJECT CLASSIFICATION CODES. 30C50, $30 \mathrm{C75}$.

1. INTRODUCTION.

Let $S$ denote the class of all functions $f(z)$ which are analytic and univalent in the unit disk $U=\{z:|z|<1\}$ and are normalized so that $f(0)=0$ and $f^{\prime}(0)=1$. The logarithmic coefficients $\gamma_{k}$ of $f(z)$ are defined by the relation

$$
\log \left(\frac{f(z)}{z}\right)=2 \sum_{k=1}^{\infty} \gamma_{k} z^{k}
$$

In particular, the Koebe function $k(z)=z(1-z)^{-2}$ has logarithmic coefficients $\gamma_{k}=1 / k(k=1,2, \ldots)$.

In fundamental work published during the 1960's, I. M. Milin concerned himself with logarithmic coefficients and their role in the theory of univalent functions. Subsequent to a great deal of intense research, Milin conjectured the inequalities stated in Theorem 1 below. These inequalities attracted much attention because their truth would imply the the truth of the Robertson conjecture and the Bleberbach conjecture, in addition to others. Then, in 1984, Louis de Branges [1, p. 146-150] proved these inequalities using Loewner's parametric method and a special system of strictly decreasing weight functions. An alternate treatment of these inequalities was subsequently presented by Fitzgerald and Pommerenke [2, pp. 683-690]. Since then, many authors have explored these inequalities. In this paper we shall also do so, but first we restate de Branges' famous result. 
THEOREM 1. (de Branges) Let $f \in S$, and let $\gamma_{k}(k=1,2, \ldots)$ denote the logarithmic coefficients of $f$. Then, for every $N \geq 1$, we have

$$
\sum_{k=1}^{N} k(N+1-k)\left|\gamma_{k}\right|^{2} \leq \sum_{k=1}^{N} \frac{N+1-k}{k} .
$$

Equality holds if and only if $f(z)=z /(1-\eta z)^{2},|\eta|=1$.

It is our purpose here to derive further consequences of Theorem 1 by utilizing the following equivalent reformulation of it.

THEOREM 1'. Let $f \in S$, and let $\gamma_{k}(k=1,2, \ldots)$ denote the logarithmic coefficients of $f$. Let $P(k)(k=1,2, \ldots)$ be a non-negative, non-increasing convex function. Assume further that the weight function $P$ is eventually identically zero; that is, that there exists an $N$ for which $P(k)=0$ whenever $k \geq N+1$. Then, for this value of $N$, we have

$$
\sum_{k=1}^{N} k P(k)\left|\gamma_{k}\right|^{2} \leq \sum_{k=1}^{N} \frac{P(k)}{k}
$$

Equality holds if and only if $f(z)=z /(1-\eta z)^{2},|\eta|=1$.

As it is pointed out in [1], Theorem 1' follows from Theorem 1 by two Abel summations (or summations by parts). Of course, Theorem 1 follows from Theorem 1' by simply choosing the weight function

$$
P(k)=\left\{\begin{aligned}
N+1-k, & k=1, \ldots, N \\
0, & k=N+1, \ldots
\end{aligned}\right.
$$

Thus, these theorems are equivalent, even though Theorem 1' appears on the surface to be more complex.

Several authors have concerned themselves with consequences of these inequalities and with other inequalities which are similar in nature. Milin and Grinshpan [3, pp.139-147] derived a necessary condition which must be satisfied by the weights $P(k)$ in Theorem 1' if the Koebe function is to be extremal, and explored some of its consequences. Andreev and Duren [3, pp. 721-728] reproved this necessary condition in a different manner, and detailed its consequences.

Many choices for $P$ yield interesting applications of this theorem. However, for some desired choices, such as $P(k)=k^{-\alpha}(\alpha>0)$, or $P(k)=r^{k}(0<r<1)$, the assumption that $P$ is eventually identically zero could never be met. The simple process of truncation produces a sequence $\{P(1), \ldots, P(N), 0,0, \ldots\}$ which is eventually identically zero, but then the truncated sequence need not be convex, thereby preventing the application of Theorem 1'.

In Section 2, we provide a simple method of circumventing this difficulty.

In Section 3, we take advantage of this method in a natural manner to obtain several interesting consequences which extend and generalize known results. 
2. REDEFINING THE CONVEX WEIGHT FUNCTION $P(k)$.

Let $N \geq 2$ be fixed, and $P(k)(k=1,2, \ldots)$ be given. Assume that $P$ is non-negative, non-increasing, and convex. Assume further that the weight function $P(k)>0$ for all $k \geq 1$ (1.e., that $P$ is not eventually identically zero), but that $P(k) \rightarrow 0$ as $k \rightarrow \infty$.

If $P(N-1)>P(N)$, then there is a unique straight line which passes through the points $(N-1, P(N-1))$ and $(N, P(N))$. The equation of this line is

$$
y=[P(N)-P(N-1)] x+[N P(N-1)-(N-1) P(N)],
$$

and the $x$-intercept of this line is easily seen to be

$$
x_{N}=N+\frac{P(N)}{P(N-1)-P(N)} .
$$

We consider two cases.

CASE I: If

$$
0<\frac{P(N)}{P(N-1)-P(N)} \leq 1 \text {, }
$$

or equivalently, if $2 P(N) \leq P(N-1)$, then we define a new convex weight function by

$$
\hat{P}(k)=\left\{\begin{aligned}
& P(k), k=1, \ldots, N \\
& 0, k \geq N+1
\end{aligned}\right.
$$

In this case, $\hat{P}$ is a simple truncation of $P$, and it is geometrically evident that $\hat{P}$ satisfies all preconditions necessary to apply Theorem $1^{\prime}$.

CASE II: If

$$
1<\frac{P(N)}{P(N-1)-P(N)}<+\infty .
$$

then we define a new convex weight function $\hat{P}$ by including a mid-range linear section, the addition of which will facilitate the estimations to follow.

Specifically, we define

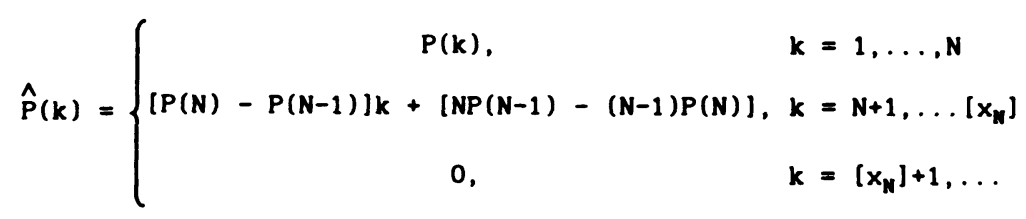

where $[$ ] denotes the greatest integer function. Since $P$ has been redefined in a linear fashion for $m i d-r a n g e$ and end-range values of $k$, it is again geometrically clear that $\hat{P}$ satisfies all pre-conditions necessary in order to apply Theorem 1'. In so doing, we obtain 


$$
\sum_{k=1}^{N} k P(k)\left|\gamma_{k}\right|^{2}+\sum_{k=N+1}^{N+N P} k \hat{P}(k)\left|\gamma_{k}\right|^{2} \leq \sum_{k=1}^{N} \frac{P(k)}{k}+\sum_{k=N+1}^{N+N P} \frac{\hat{P}(k)}{k}
$$

where we have set $N_{P}=[P(N) /(P(N-1)-P(N))]$.

This last inequality allows for convenient estimations. For example, if we set $\hat{P}(k)=a k+b$, where $a=P(N)-P(N-1)$ and $b=N P(N-1)-(N-1) P(N)$, then

$$
\sum_{k=N+1}^{N+N_{P}} \frac{\hat{P}(k)}{k}=\sum_{k=N+1}^{N+N_{P}} \frac{a k+b}{k}<a N_{P}+b \ln \left(1+\frac{N_{P}}{N}\right)
$$

Hence, estimates of the form

$$
\sum_{k=1}^{N} k P(k)\left|\gamma_{k}\right|^{2}<\sum_{k=1}^{N} \frac{P(k)}{k}+a N_{P}+b \ln \left(1+\frac{N_{P}}{N}\right)
$$

are possible, with no explicit reference to the values of $\hat{P}$. Also, since we have constructed $\hat{P}(k) \leq P(k)$, we obtain the inequalities

$$
\sum_{k=1}^{N} k P(k)\left|\gamma_{k}\right|^{2} \leq \sum_{k=1}^{N+N_{P}} \frac{P(k)}{k} \leq \sum_{k=1}^{\infty} \frac{P(k)}{k}
$$

so that

$$
\sum_{k=1}^{\infty} k P(k)\left|\gamma_{k}\right|^{2} \leq \sum_{k=1}^{\infty} \frac{P(k)}{k}
$$

which is significant whenever the series on the right converges.

The above observations were made under the assumption that $P(N-1)>P(N)$. If $\mathrm{P}(\mathrm{N}-1)=\mathrm{P}(\mathrm{N})>0$, then the convexity condition would require that $\mathrm{P}(\mathrm{k})=$ $P(N)$ for all $k>N$. But then $P$ could never be redefined as above to be eventually identically zero. In addition, the infinite series on the right side of (2.1) would diverge by comparison to the harmonic series. Such a cholce for $P$ would be inadmissible.

of course, every admissible choice of $P$ yields a new inequality of the form (2.1). In the next section, we present some of these choices.

3. RESULTS.

Andreev and Duren [3, p. 722] asked whether the partial sum inequality

$$
\sum_{k=1}^{N} k\left|\gamma_{k}\right|^{2} r^{k} \leq \sum_{k=1}^{N} \frac{1}{k} r^{k}
$$

holds for any $r>0$. Although they provide some supportive evidence that this inequality should hold for $r \leq 1 / 2$, they also establish [3, p. 727] that for no $\mathrm{N} \geq 2$ can it hold for all $r<1$. 
In Theorem 2(a) below, we shall show that this inequality does indeed hold if $r \leq 1 / 2$. It is not known whether the constant " $1 / 2$ " is best possible. Within the proof of Theorem 2(b), a method of estimating the left-hand side of the above inequality when $1 / 2<r<1$ is provided. Although Theorem $2(b)$ has been obtained elsewhere (see, for example, Milin and Grinshpan [3, p. 143]), our purpose here is to show that it may be easily obtained from Theorem 1'.

THEOREM 2. Let $f \in S$, and let $\gamma_{k}(k=1,2, \ldots)$ denote the logarithmic coefficients of $f$.

(a) If $0 \leq r \leq 1 / 2$, then for every $N \geq 1$, we have

$$
\sum_{k=1}^{N} k\left|\gamma_{k}\right|^{2} r^{k} \leq \sum_{k=1}^{N} \frac{1}{k} r^{k}
$$

Equality holds if and only if $f(z)=z /(1-\eta z)^{2},|\eta|=1$.

(b) For each $r, 0 \leq r<1$,

$$
\sum_{k=1}^{\infty} k\left|\gamma_{k}\right|^{2} r^{k} \leq \sum_{k=1}^{\infty} \frac{1}{k} r^{k}=\ln \left(\frac{1}{1-r}\right)
$$

PROOF. For $0 \leq r \leq 1 / 2$, the weight function defined by

$$
\hat{P}(k)=\left\{\begin{aligned}
r^{k}, & k=1, \ldots, N \\
0, & k=N+1, \ldots
\end{aligned}\right.
$$

is convex. Hence, inequality (3.1) follows immediately from Theorem 1'.

(b) If $1 / 2<r<1$, then there exists a unique positive integer $J$ such that $j /(j+1)<r \leq(j+1) /(j+2)$. For this value of $j$ and any $N \geq 1$, we define

$$
\hat{P}(k)=\left\{\begin{aligned}
r^{k}, & k=1, \ldots, N \\
\left(r^{N}-r^{N-1}\right) k+\left(N r^{N-1}-(N-1) r^{N}\right), & k=N+1, \ldots, N+j \\
0, & k=N+j+1, \ldots
\end{aligned}\right.
$$

Applying Theorem 1', we now obtain

$$
\sum_{k=1}^{N} k\left|\gamma_{k}\right|^{2} r^{k}+\sum_{k=N+1}^{N+j} k \hat{P}(k)\left|\gamma_{k}\right|^{2} \leq \sum_{k=1}^{N} \frac{r^{k}}{k}+\sum_{k=N+1}^{N+j} \frac{\hat{P}(k)}{k} .
$$

For $1 / 2<r<1$, the left-hand side of (3.1) may now be estimated by ignoring the second term on the left-hand side of (3.2), and substituting the values of $\hat{P}(k)$ on the right. The calculations involved in this estimation may be eased by noting that $\hat{P}(k) \leq r^{k}$ for mid-range values of $k$.

The result of part (b) now follows by letting the index $N \rightarrow \infty$ in (3.2) 
Theorem 3 to follow is a generalization of the result

$$
\sum_{k=1}^{\infty}\left|\gamma_{k}\right|^{2} \leq \sum_{k=1}^{\infty} \frac{1}{k^{2}}=\frac{\pi^{2}}{6}
$$

which was previously established by Duren and Leung [5, pp. 36-43]. Of course, the Koebe function is extremal for this inequality, since its coefficients are precisely $\gamma_{k}=1 / k(k=1,2, \ldots)$. In Corollary 3.1, we obtain an estimate on the $N^{\text {th }}$ partial sums of this series, which is valid for $N \geq 3$.

THEOREM 3. Let $f \in S$, and let $\gamma_{k}(k=1,2, \ldots)$ be the logarithmic coefficlents of $f$.

(a) If $\alpha>0$ and $N \leq 1+\frac{1}{2^{1 / \alpha}-1}$, then

$$
\sum_{k=1}^{N} k^{1-\alpha}\left|\gamma_{k}\right|^{2} \leq \sum_{k=1}^{N} \frac{1}{k^{1+\alpha}}
$$

Equality holds if and only if $f(z)=z /(1-\eta z)^{2},|\eta|=1$.

(b) If $\alpha>0$, then

$$
\sum_{k=1}^{\infty} k^{1-\alpha}\left|\gamma_{k}\right|^{2} \leq \sum_{k=1}^{\infty} \frac{1}{k^{1+\alpha}}=\zeta(1+\alpha)
$$

where $\zeta$ denotes the Riemann Zeta Function.

PROOF. (a) For $\mathrm{N}$ and $\alpha$ in this range, the weight function defined by

$$
\hat{P}(k)=\left\{\begin{aligned}
k^{-\alpha}, k & =1, \ldots, N \\
0, k & =N+1, \ldots
\end{aligned}\right.
$$

is convex. Hence, (3.3) follows directly from Theorem 1'.

(b) If $\mathrm{N}>1+1 /\left(2^{1 / \alpha}-1\right)$, then there exists a unique integer $\mathrm{J}$ such that

$$
j<\frac{(N-1)^{\alpha}}{N^{\alpha}-(N-1)^{\alpha}} \leq j+1 .
$$

For these values of $N, j$, and $\alpha$, we define

$$
\hat{P}(k)=\left\{\begin{array}{cl}
k^{-\alpha}, & k=1, \ldots, N \\
\left(N^{-\alpha}-(N-1)^{-\alpha}\right) k+\left(N(N-1)^{-\alpha}-(N-1) N^{-\alpha}\right), & k=N+1, \ldots, N+j \\
0, & k=N+J+1, \ldots
\end{array}\right.
$$

Applying Theorem 1', we now obtain

$$
\sum_{k=1}^{N} k^{1-\alpha}\left|\gamma_{k}\right|^{2}+\sum_{k=N+1}^{N+j} \hat{k P}(k)\left|\gamma_{k}\right|^{2} \leq \sum_{k=1}^{N} \frac{1}{k^{1+\alpha}}+\sum_{k=N+1}^{N+1} \frac{\hat{P}(k)}{k}
$$


Since $\hat{P}(k) \leq 1 / k^{\alpha}$, estimates of the form

$$
\sum_{k=1}^{N} k^{1-\alpha}\left|\gamma_{k}\right|^{2} \leq \sum_{k=1}^{N+j} \frac{1}{k^{1+\alpha}}
$$

are now possible, where $j$ is defined as above.

The conclusion of part (b) now follows by letting the index $N \rightarrow \infty$ in the inequality stated above.

In the following corollary, we consider the Duren-Leung conjecture which states that

$$
\sum_{k=1}^{N}\left|\gamma_{k}\right|^{2} \leq \sum_{k=1}^{N} \frac{1}{k^{2}}
$$

for all $N \geq 3$. Both Milin and Grinshpan [4, p. 144] as well as Andreev and Duren [3, p. 726] provide supportive evidence for this conjecture, although neither provides a proof. In the event that this conjecture is false, it would be advantageous to have an accurate estimate of the left-hand side.

COROLLARY 3.1. Let $f \in S$, and let $\gamma_{k}(k=1,2, \ldots)$ be the logarithmic coefficients of $f$. Then, for every $N \geq 3$, we have

$$
\sum_{k=1}^{N}\left|\gamma_{k}\right|^{2} \leq \sum_{k=1}^{N} \frac{1}{k^{2}}+\frac{N-2}{2 N(N-1)}
$$

PROOF. For $\mathbf{N} \geq 3$, we define $\hat{P}(k)$ as in the proof of Theorem $3(b)$ which, after some simplification, becomes

$$
\hat{P}(k)=\left\{\begin{array}{cl}
1 / k, & k=1, \ldots, N \\
\frac{2 N-k-1}{N(N-1)}, & k=N+1, \ldots, 2 N-2 \\
0, & k=2 N-1, \ldots
\end{array}\right.
$$

After applying Theorem 1', we obtain

$$
\sum_{k=1}^{N}\left|\gamma_{k}\right|^{2}+\sum_{k=N+1}^{2 N-2} \frac{k(2 N-k-1)}{N(N-1)}\left|\gamma_{k}\right|^{2} \leq \sum_{k=1}^{N} \frac{1}{k^{2}}+\sum_{k=N+1}^{2 N-2} \frac{(2 N-k-1)}{k N(N-1)}
$$

Since $\hat{P}(k) \leq 1 / k$ for $k=N+1, \ldots 2 N-2$ (this is geometrically clear), we may continue our estimation to conclude that

$$
\sum_{k=1}^{N}\left|\gamma_{k}\right|^{2}<\sum_{k=1}^{N} \frac{1}{k^{2}}+\sum_{k=N+1}^{2 N-2} \frac{1}{k^{2}}
$$

Finally, an integral estimate of the term on the right finishes the proof. 


\section{ACKNOWLEDGEMENT}

The author wishes to recognize the referee for several helpful comments in his review of this article.

\section{REFERENCES}

1. DE BRANGES, L. A Proof of the Bieberbach Conjecture, Acta Math. 128(1985), 137152.

2. FITZGERALD, C. and POMMERENKE, Ch. The de Branges Theorem on Univalent Functions, Trans. Am. Math. Soc. $90(2)(1985), 683-690$.

3. ANDREEV, V.V. and DUREN, P.L. Inequalities for Logarithmic Coefficients of Univalent Functions and their Derivatives, Indiana Univ. Math. J. 37(4)1988, 721733.

4. MILIN, I.M. and GRINSHPAN, A.Z. Logarithmic Coefficient Means of Univalent Functions, Complex Variables Theory Appl. Z(1986), 139-147.

5. DUREN, P.L. and LEUNG, Y.J. Logarithmic Coefficients of Univalent Functions, J. d'Analyse Math. 36(1979), 36-43. 


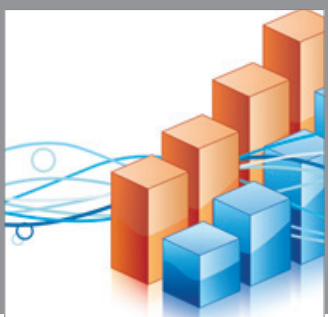

Advances in

Operations Research

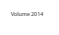

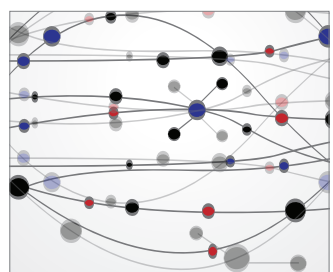

\section{The Scientific} World Journal
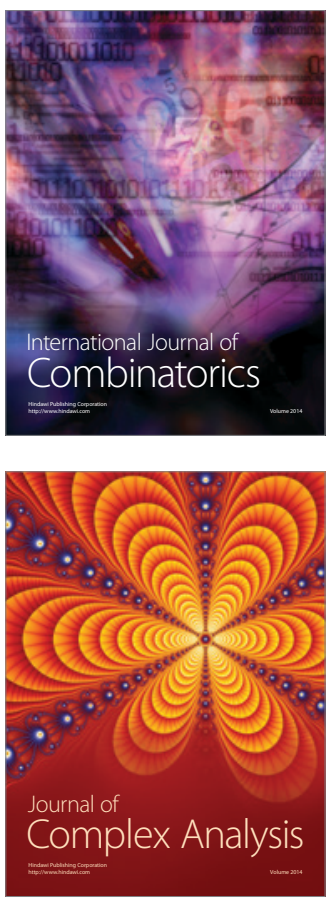

International Journal of

Mathematics and

Mathematical

Sciences
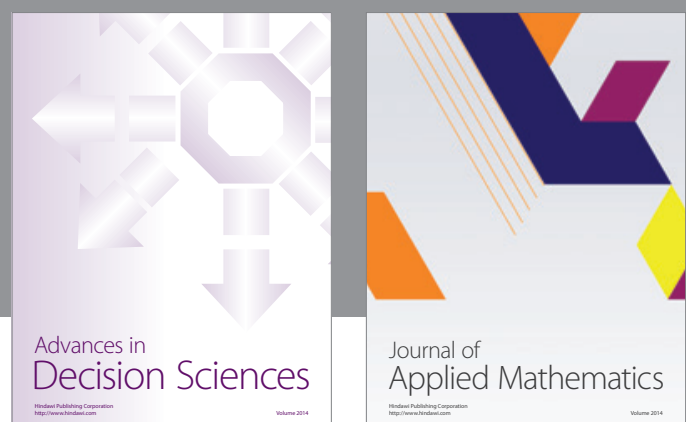

Journal of

Applied Mathematics
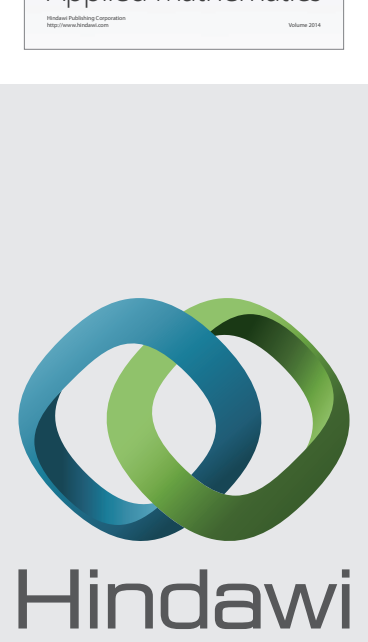

Submit your manuscripts at http://www.hindawi.com
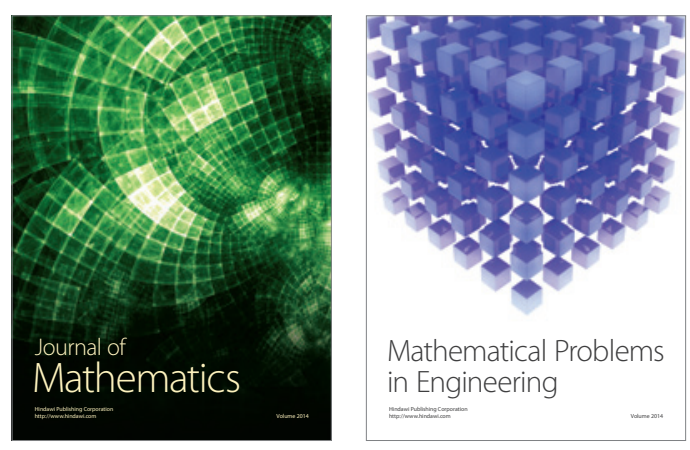

Mathematical Problems in Engineering
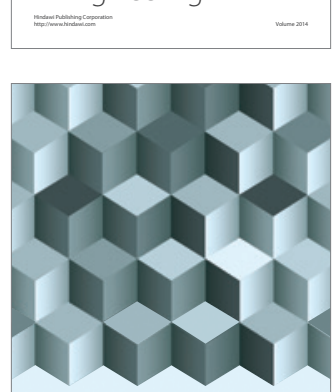

Journal of

Function Spaces
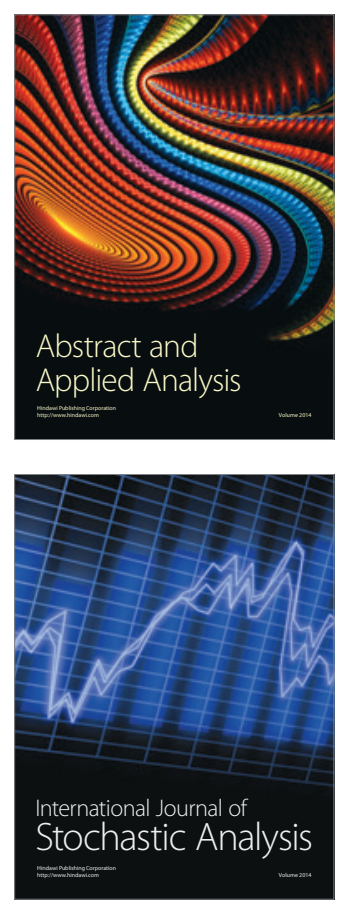

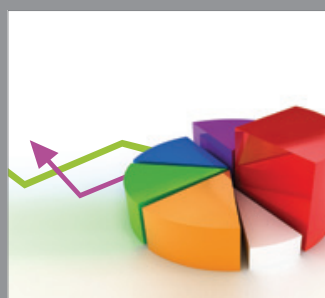

ournal of

Probability and Statistics

Promensencen
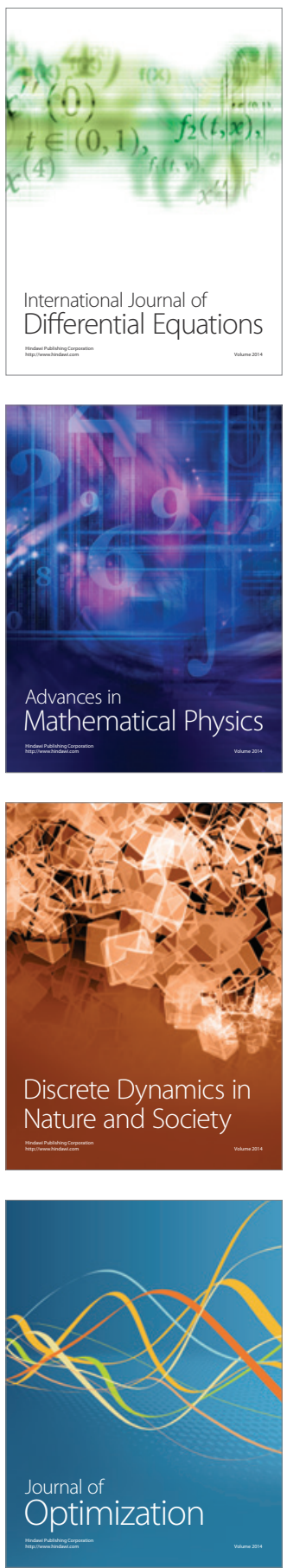